\title{
DECOMPOSIÇÃO DO METANO EM CATALISADORES DE COBALTO SUPORTADOS - O EFEITO DAS PROPRIEDADES TEXTURAIS NA ATIVIDADE CATALÍTICA PARA FORMAÇÃO DE CARBONO FILAMENTAR
}

\author{
Rosenir Rita de Cassia Moreira da Silva! \\ Bárbara Thomazelli Marques de Oliveira ${ }^{1}$ \\ Ana Carolina Pietro Fernandes Guarino ${ }^{1}$, \\ Fábio Barboza Passos ${ }^{1}$
}

\begin{abstract}
Resumo: Atualmente, existem muitos estudos sendo realizados visando à formação de materiais nanoestruturados. A formação dessas estruturas ocorre através de reação química em superfícies metálicas, principalmente contendo níquel e cobalto. Neste trabalho, foram preparados catalisadores de cobalto, suportados em $\mathrm{SiO}_{2}, \mathrm{Al}_{2} \mathrm{O}_{3}$ e $\mathrm{Nb}_{2} \mathrm{O}_{5}$ para serem testados na decomposição do $\mathrm{CH}_{4}$ com produção de $\mathrm{H}_{2}$ e formação de depósitos carboníferos. Sabe-se que o tamanho das partículas metálicas, sua dispersão, área específica e estrutura dos suportes influenciam bastante o processo. Diversas técnicas foram utilizadas para caracterizar os catalisadores: determinação da área específica (BET); difração de raio $\mathrm{X}$ (DRX), que mostrou a presença da fase cristalina dos suportes; quimissorção de hidrogênio, para quantificação de sítios de cobalto metálico dispersos na superfície, e redução à temperatura programada (TPR) para avaliar a redutibilidade das espécies de cobalto. Os tamanhos médios das partículas de cobalto também puderam ser estimados pela quimissorção. Foram realizados ainda testes catalíticos, que permitiram avaliar a reatividade para decomposição do metano e a formação dos depósitos carboníferos nos catalisadores metálicos, sendo que os catalisadores de $\mathrm{Co} / \mathrm{SiO}_{2}$ reduzidos a $500^{\circ} \mathrm{C}$ exibiram as maiores conversões.
\end{abstract}

Palavras chaves: decomposição do metano, nanoestruturas de carbono, catalisadores de cobalto suportados.

\begin{abstract}
Now a day, numerous investigations have been made to study nanostructures materials formations. These structures can be formed by chemical reactions on metallic surfaces, especially nickel and cobalt. In this work, cobalt catalysts supported on $\mathrm{SiO}_{2}$, $\mathrm{Al}_{2} \mathrm{O}_{3}$ and $\mathrm{Nb}_{2} \mathrm{O}_{5}$ were prepared to be tested for methane decomposition with $\mathrm{H}_{2}$ and carbon filaments productions. It seems that metallic particles sizes, there dispersions on support, the surface area and supports structures strongly influence this process. BET, X ray diffraction (XRD), hydrogen chemisorption and temperature programmed reduction (TPR) techniques were used to characterize catalysts surface. Average metal particles sizes of cobalt catalysts could be calculated by chemisorption measures. The catalytic measurements were performed in order to determine the catalytic activity for the decomposition of methane, with carbon deposits formations. The results indicate that cobalt catalysts supported on silica reduced at $500^{\circ} \mathrm{C}$ exhibit the most conversions.
\end{abstract}

Keywords: Methane decomposition, carbon nanostructures, cobalt supported catalysts

${ }^{1}$ Departamento de Engenharia Química e de Petróleo - UFF - rosenir@vm.uff.br 


\section{INTRODUÇÃO}

A conversão do metano tem adquirido grande relevância nos últimos anos devido à enorme produção de gás natural no mundo, sendo metano o seu principal constituinte. Pode-se destacar a utilização do gás natural como fonte de energia, principalmente como combustível, e como fonte de matériaprima, para produção de diversas substâncias para a indústria química e para a fabricação de novos materiais.

O acoplamento não oxidativo, em dois estágios, utilizando catalisadores de $\mathrm{Co}, \mathrm{Ni}, \mathrm{Pd}$ e $\mathrm{Ru}$, suportados em diversos óxidos $\left(\mathrm{Al}_{2} \mathrm{O}_{3}, \mathrm{SiO}_{2}, \mathrm{CeO}_{2}, \mathrm{ZrO}_{2}\right.$, etc. $)$, tem sido testado como rota para transformar o metano em hidrocarbonetos líquidos de peso molecular mais elevados (Adveeva et al., 1999, Zadeh e Smith, 1998, Guarino, 2005, Ferreira-Aparício et al., 1997, Li et al., 2006, Boskovik e Smith, 1997, Ogihara et al., 2006). Nesta rota, num primeiro estágio, ocorre a decomposição do metano gerando hidrogênio e depósitos de carbono $\left(\mathrm{CH}_{4}\right.$ $\rightarrow 2 \mathrm{H}_{2}+\mathrm{C}$ ) e num segundo estágio, ocorre a hidrogenação deste carbono em baixas temperaturas, formando hidrocarbonetos de pesos moleculares mais elevados.

Considerando o primeiro estágio, os produtos desta reação possuem importantes aplicações, pois além do hidrogênio, que poderia ser utilizado em células de combustíveis, pode-se, dependendo das condições reacionais e dos catalisadores empregados, produzir carbono filamentar ou nanotubos de carbono, este último de grande interesse industrial, por possuir propriedades eletrônicas e mecânicas especiais. Nos nanotubos de carbono, as ligações químicas entre os átomos de carbono (ligações covalentes) são muito fortes, conferindo à essa estrutura grande estabilidade e resistência mecânica, inclusive a campos elétricos externos.

O mecanismo de formação e crescimento dos depósitos de carbono tem sido bastante estudado utilizando-se catalisadores de Ni (Ermakova et al., 2000, Solymosi et al., 1994, Figueiredo et al., 1989). Os catalisadores de cobalto produzem outros tipos de filamentos de carbono, quando comparados com os catalisadores de níquel, formando diferentes morfologias, com diferentes propriedades tecnológicas. Avdeeva e colaboradores (1999) em seus estudos verificaram a formação de uma morfologia do tipo núcleo oco. A Figura 1(a) e 1(b) apresenta algumas dessas morfologias também obtidas a partir da decomposição do CO (Pinheiro et al., 2002).

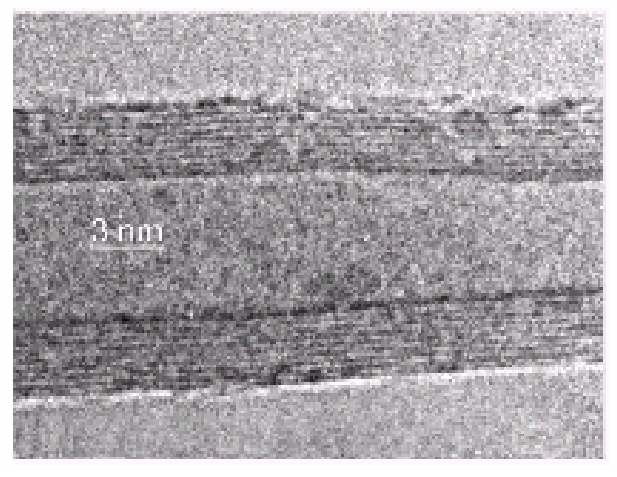

(a)

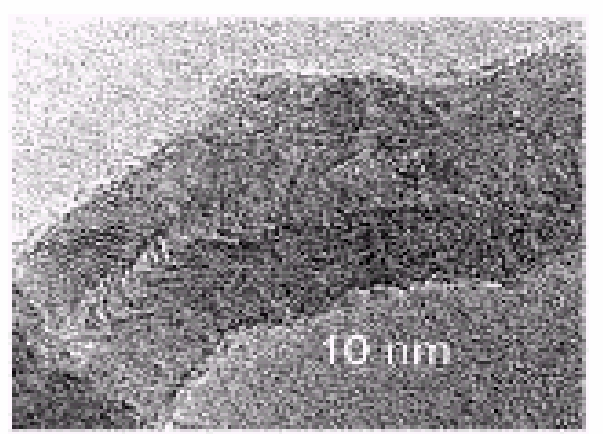

(b)

Figura 1 - Depósitos carboníferos formados a partir da decomposição do $\mathrm{CO}$ em catalisadores de $\mathrm{Co} / \mathrm{Al}_{2} \mathrm{O}_{3}$. (a) Nanotubos de carbono formados a partir da reação com CO puro. (b) Filamentos de carbono formados a partir da reação $\mathrm{CO}$ com 5\% de $\mathrm{H}_{2}$ (Pinheiro et al., 2002)

O mecanismo proposto para a reação considera a migração de espécies de carbono adsorvidas no metal para o suporte (Adveeva et al., 1999, Solymosi et al., 1993, Ferreira-Aparício et al., 1997, Martins e Schmal, 2006) e o modelo cinético, desenvolvido para descrever a reação de decomposição do metano, em catalisadores à base de cobalto, supõe a decomposição do $\mathrm{CH}_{4}$ 
num sítio de Co metálico produzindo $\mathrm{H}_{2}$ e depósitos carboníferos, seguida da possível migração de parte das espécies $\mathrm{CH}_{\mathrm{x}}$, adsorvidas na superfície do Co, para o suporte (Zhang e Smith, 2002, 2005, Martins e Schmal, 2006) . Diversos fatores podem afetar a taxa de decomposição do metano e a migração dessas espécies na superfície, tais como a temperatura da reação, o teor metálico do catalisador, o tamanho das partículas metálicas e o tipo de suporte.

O presente trabalho tem por objetivo verificar a influência das propriedades texturais de catalisadores de cobalto suportados em sílica, alumina e nióbia, na reação de decomposição do metano, a fim de se investigar a atividade catalítica para geração de hidrogênio e produção de depósitos carboníferos. Uma vez que os metais do grupo VIII suportados em $\mathrm{Nb}_{2} \mathrm{O}_{5}$ podem apresentar forte interação metal-suporte, o efeito SMSI (Strong Metal Support Interaction), quando reduzidos a altas temperaturas (Silva et al., 1993, Lapidus et. al, 1991), que consiste na redutibilidade parcial do suporte produzindo espécies $\mathrm{NbO}_{\mathrm{x}}$ movéis que migrariam para a superfície das partículas metálicas, encobrindo-as, provocando queda na atividade catalítica, pretende-se avaliar a reação na ausência e presença de tal efeito.

\section{EXPERIMENTAL}

\section{Preparação dos Catalisadores}

Os materiais de partida utilizados para a obtenção dos suportes $\mathrm{SiO}_{2}, \gamma$ $\mathrm{Al}_{2} \mathrm{O}_{3}$ e $\mathrm{Nb}_{2} \mathrm{O}_{5}$ foram a $\mathrm{SiO}_{2}$ (Davicat SP 550-10022), a bohemita (Catapal) e o ácido nióbico (HY 340 CBMM), respectivamente. Esses materiais foram calcinados a $550^{\circ} \mathrm{C}$ por $2 \mathrm{~h}$ em mufla. Os catalisadores foram preparados pelo método de impregnação a seco, utilizando $\mathrm{Co}\left(\mathrm{NO}_{3}\right)_{2} \cdot 6 \mathrm{H}_{2} \mathrm{O}$, fornecido pela MERCK, como precursor, com teores que variaram de $5 \%$ a $20 \%$ em massa de cobalto, para os catalisadores suportados em sílica, e com teor de $10 \%$ para os suportados em alumina e nióbia.
Após a impregnação, todas as amostras foram secas em estufa a $120^{\circ} \mathrm{C}$ por $24 \mathrm{~h}$, e calcinadas em mufla a $400^{\circ} \mathrm{C}$ por 2 horas.

\section{Caracterização dos Catalisadores}

Diversas técnicas foram utilizadas para caracterizar a superfície dos catalisadores. As áreas específicas e os volumes de poros foram determinados utilizando-se um aparelho automático ASAP (Accelerated Surface Área and Porosimetry System) $2010 \quad$ da Micromeritics.

Os experimentos de TPR (Redução

à Temperatura Programada) foram realizados em uma unidade multipropósito acoplada a um espectrômetro de massas Balzers Omnistar. O pré-tratamento das amostras consistiu na secagem a $150^{\circ} \mathrm{C}$, por 30 min, sob fluxo de $\mathrm{He}(30 \mathrm{~mL} / \mathrm{min})$, seguida de redução sob fluxo de $5 \%$ $\mathrm{H}_{2}$ /Argônio a uma velocidade de aquecimento de $10^{\circ} \mathrm{C} / \mathrm{min}$ até $800^{\circ} \mathrm{C}$ por 2h.

As medidas de quimissorção de $\mathrm{H}_{2}$ foram feitas utilizando-se o equipamento ASAP 2010 da Micromeritics. Através dessas medidas é possível quantificar o número de sítios ativos constituídos de cobalto metálico dispersos na superfície do suporte após a ativação do catalisador, uma vez que o cobalto metálico adsorve quimicamente o hidrogênio. Uma massa da amostra de aproximadamente $1,5 \mathrm{~g}$ do catalisador previamente calcinada foi colocada no reator da unidade. Em seguida, procedeu-se à limpeza da amostra em vácuo para então se realizar a ativação "in situ” com $\mathrm{H}_{2}$ puro, nas temperaturas de $300^{\circ} \mathrm{C}$, por $3 \mathrm{~h}$, e de $500^{\circ} \mathrm{C}$, por $2 \mathrm{~h}$, para todos os catalisadores. Após essas etapas foram determinadas as isotermas de quimissorção de $\mathrm{H}_{2}$. A seqüência empregada para o pré-tratamento da amostra, redução e análise consistiu em:

i. Aquecer a amostra a uma taxa de $10^{\circ} \mathrm{C} / \mathrm{min}$ até $120^{\circ} \mathrm{C}$, com fluxo de $\mathrm{H}_{2}$, e manter por $30 \mathrm{~min}$;

ii. Fazer vácuo na amostra por $60 \mathrm{~min}$, a $120^{\circ} \mathrm{C}$, até vácuo de $10-5$ Torr; 
iii. Realizar teste de vazamento nesta temperatura;

iv. Proceder a redução com $\mathrm{H}_{2}$ a $300^{\circ} \mathrm{C}$ ou $500^{\circ} \mathrm{C}$ (com taxa de $10^{\circ} \mathrm{C} / \mathrm{min}$ ), por 3 ou 2h, respectivamente.

v. Fazer vácuo por $1 \mathrm{~h}$ na temperatura de redução;

vi. Proceder à análise a $150^{\circ} \mathrm{C}$ traçando a curva de isoterma de adsorção total de $\mathrm{H}_{2}$ até pressões de $300 \mathrm{mmHg}$. Buscou-se ajustar os tempos de equilíbrio e as tolerâncias relativas para todas as amostras.

vii. Fazer vácuo por $1 \mathrm{~h}$ na temperatura da análise da primeira isoterma, $150^{\circ} \mathrm{C}$;

viii. Proceder à análise a $150^{\circ} \mathrm{C}$ traçando a curva de isoterma de adsorção reversível de $\mathrm{H}_{2}$ até pressões $300 \mathrm{mmHg}$.

\section{Reação de decomposição do metano}

Os experimentos foram realizados numa unidade convencional, munida de um espectrômetro de massas, acoplados a um cromatógrafo a gás para análise em linha dos produtos formados. O prétratamento das amostras consistiu na secagem a $150^{\circ} \mathrm{C}$, por 30 min sob fluxo de He $(30 \mathrm{~mL} / \mathrm{min})$, seguida de redução sob fluxo de $\mathrm{H}_{2}$ (vazão de $100 \mathrm{ml} / \mathrm{min}$ ) a diferentes temperaturas, $500^{\circ} \mathrm{C}$ por $2 \mathrm{~h}$ e $300^{\circ} \mathrm{C}$ por $3 \mathrm{~h}$, a fim de se verificar a influência da redutibilidade do catalisador. A seguir, as amostras foram resfriadas ou aquecidas, dependendo da temperatura de redução, até $450^{\circ} \mathrm{C}$ para então iniciar a reação de decomposição sob fluxo de $20 \% \mathrm{CH}_{4} / \mathrm{He}$ a uma vazão de $100 \mathrm{ml} / \mathrm{min}$.

\section{RESULTADOS}

\section{1. ÁREA ESPECÍFICA (BET) E VOLUME DE POROS}

A Tabela 1 apresenta as áreas específicas e os volumes de poros dos catalisadores e suportes utilizados. Podese observar que a sílica e a alumina apresentam uma grande área específica, enquanto a nióbia apresenta uma pequena área. Na distribuição de volume de poros da sílica e da alumina detectou-se uma estrutura mais microporosa, enquanto a nióbia apresenta uma estrutura mais macroporosa. Após a impregnação do metal nos suportes houve uma pequena diminuição nas áreas específicas dos catalisadores correspondentes às variações dos teores metálicos.

Tabela 1. Área BET e Volume de poros dos suportes e catalisadores $\mathrm{Co} / \mathrm{SiO}_{2}, \mathrm{Co} / \gamma$ $\mathrm{Al}_{2} \mathrm{O}_{3}$ e $\mathrm{Co} / \mathrm{Nb}_{2} \mathrm{O}_{5}$

\begin{tabular}{|l|c|c|}
\hline Suportes e Catalisadores & $\begin{array}{c}\text { Área BET } \\
\left(\mathbf{m}^{2} / \mathbf{g}\right)\end{array}$ & $\begin{array}{c}\text { Volume de poros } \\
\left(\mathbf{c m}^{\mathbf{3}} / \mathbf{g}\right)\end{array}$ \\
\hline $\mathrm{SiO}_{2}$ calc. a $550^{\circ} \mathrm{C}$ & 417 & 1,518 \\
\hline$\gamma$ - $\mathrm{Al}_{2} \mathrm{O}_{3}$ calc. a $550^{\circ} \mathrm{C}$ & 271 & 0,606 \\
\hline $\mathrm{Nb}_{2} \mathrm{O}_{5}$ calc. a $550^{\circ} \mathrm{C}$ & 32,5 & 0,186 \\
\hline $5 \% \mathrm{Co} / \mathrm{SiO}_{2}$ & 382,3 & 1,402 \\
\hline $10 \% \mathrm{Co} / \mathrm{SiO}_{2}$ & 351,3 & 1,258 \\
\hline $20 \% \mathrm{Co} / \mathrm{SiO}_{2}$ & 291,5 & 1,038 \\
\hline $10 \% \mathrm{Co} / \mathrm{Al}_{2} \mathrm{O}_{3}$ & 226,6 & 0,477 \\
\hline $10 \% \mathrm{Co} / \mathrm{Nb}_{2} \mathrm{O}_{5}$ & 27,4 & 0,137 \\
\hline
\end{tabular}




\subsection{DIFRAÇÃO DE RAIO X (DRX)}

Os espectros de DRX obtidos para os suportes, catalisadores e $\mathrm{Co}_{3} \mathrm{O}_{4}$ mássico estão apresentados na Figura 2.

O DRX confirmou que o cobalto estava presente como $\mathrm{Co}_{3} \mathrm{O}_{4}$ nos catalisadores calcinados a $400^{\circ} \mathrm{C}$ e nenhuma outra fase contendo cobalto foi detectada.

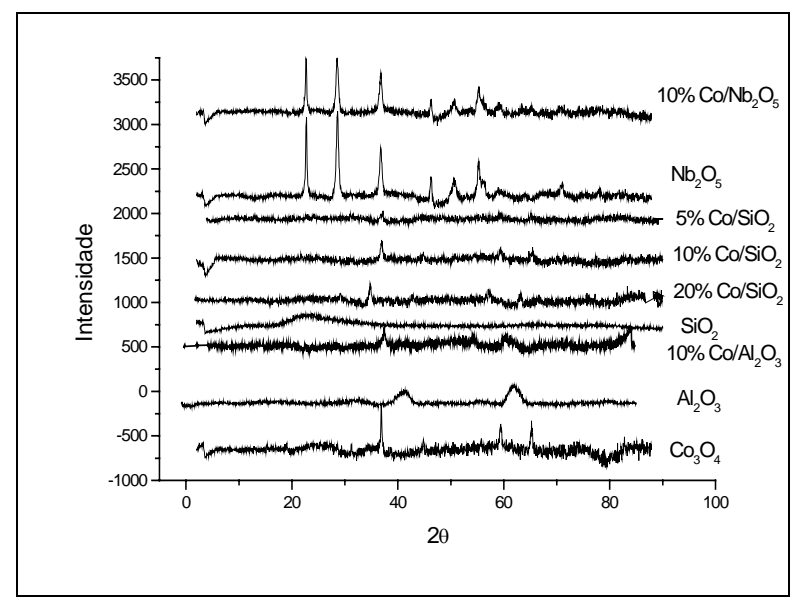

Figura 2 - Espectros de DRX obtidos para os suportes e catalisadores $\mathrm{Co} / \mathrm{SiO}_{2}$, $\mathrm{Co} / \gamma-\mathrm{Al}_{2} \mathrm{O}_{3}$ e $\mathrm{Co} / \mathrm{Nb}_{2} \mathrm{O}_{5}$ e para o $\mathrm{Co}_{3} \mathrm{O}_{4}$ mássico

Os difratogramas dos suportes permitiram identificar suas diferentes estruturas cristalinas como pode ser visto pela Tabela 2. Estes resultados confirmam a estrutura da $\gamma-\mathrm{Al}_{2} \mathrm{O}_{3}$ e a fase TT do $\mathrm{Nb}_{2} \mathrm{O}_{5}$.

Comparando o difratograma do $10 \%$ e $20 \% \mathrm{Co} / \mathrm{SiO}_{2}$ com o do $\mathrm{Co}_{3} \mathrm{O}_{4}$ é possível detectar claramente os picos do óxido de cobalto cristalino, sendo o pico de maior intensidade em $2 \theta$ igual a $36,8^{\circ}$ (100\%) em ambos os difratogramas. É observado o mesmo comportamento para o catalisador $10 \% \mathrm{Co} / \mathrm{Al}_{2} \mathrm{O}_{3}$.

No entanto, por comparação do difratograma do $10 \% \mathrm{Co} / \mathrm{Nb}_{2} \mathrm{O}_{5}$ com o do $\mathrm{Co}_{3} \mathrm{O}_{4}$, não foi possível detectar os picos do óxido de cobalto cristalino, visto que o pico de maior intensidade do mesmo localiza-se em $2 \theta$ igual a $36,8^{\circ}(100 \%)$, coincidindo com o terceiro pico da Niobia, em $2 \theta$ igual a $35,8^{\circ}$ (53\%), o que dificulta a detecção.
Tabela 2 - Sistemas Cristalinos dos suportes $\mathrm{SiO}_{2}, \gamma-\mathrm{Al}_{2} \mathrm{O}_{3}$ e $\mathrm{Nb}_{2} \mathrm{O}_{5}$ e para o $\mathrm{Co}_{3} \mathrm{O}_{4}$ mássico.

\begin{tabular}{|c|c|c|c|c|}
\hline \multicolumn{5}{|c|}{ Difraçao de Raio X - DRX } \\
\hline \hline & Lambda & Sistema & a & c \\
\hline \hline $\mathrm{SiO}_{2}$ & 1,5418 & Hexagonal & 9.92 & 81.5 \\
\hline $\mathrm{Al}_{2} \mathrm{O}_{3}$ & 1,5418 & Cúbico & 7.924 & - \\
\hline $\mathrm{Nb}_{2} \mathrm{O}_{5}$ & 1,5418 & Hexagonal & 3.607 & 3.925 \\
\hline $\mathrm{CO}_{3} \mathrm{O}_{4}$ & 1,5406 & Cúbico & 80.837 & - \\
\hline
\end{tabular}

\subsection{QUIMISSORÇÃO DE $\mathrm{H}_{2}$}

As figuras 3 e 4 apresentam os gráficos contendo as isotermas de quimissorção de $\mathrm{H}_{2}$ para os catalisadores $10 \% \mathrm{Co} / \mathrm{SiO}_{2}$ e $10 \% \mathrm{Co} / \mathrm{Nb}_{2} \mathrm{O}_{5}$.

Observa-se que após redução a $300^{\circ} \mathrm{C}$ os catalisadores adsorvem uma certa quantidade de $\mathrm{H}_{2}$, conforme pode ser verificado pelas isotermas de adsorção total e reversível (fracamente adsorvido). No entanto, após redução a $500^{\circ} \mathrm{C}$ o catalisador $10 \% \mathrm{Co} / \mathrm{Nb}_{2} \mathrm{O}_{5}$ não apresentou quimissorção de $\mathrm{H}_{2}$ mensurável, enquanto que para o catalisador $10 \% \quad \mathrm{Co} / \mathrm{SiO}_{2}$ observou-se apenas uma queda, compatível com uma sinterização.

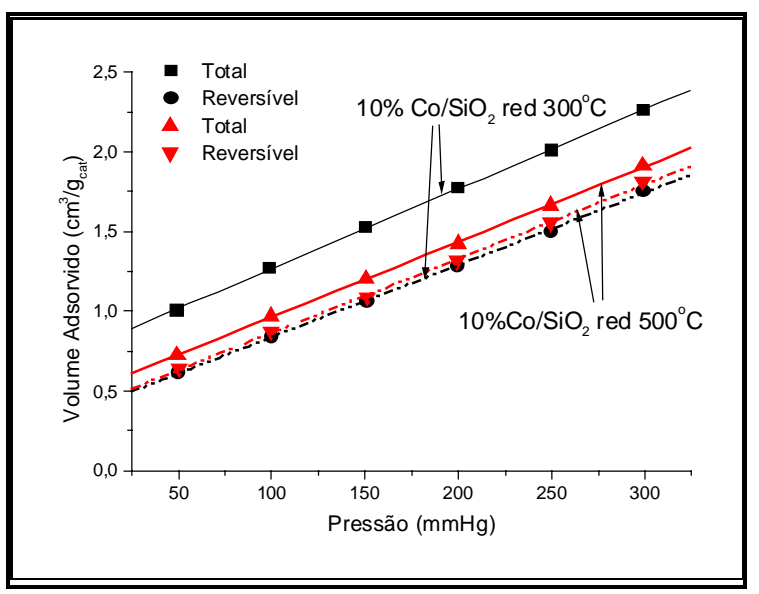

Figura 3 - Quimissorção de $\mathrm{H}_{2}$ para o catalisador $10 \% \quad \mathrm{Co} / \mathrm{SiO}_{2}$ reduzido a $300^{\circ} \mathrm{C}$ e a $500^{\circ} \mathrm{C}$

Os resultados estão sumarizados na Tabela 3, que apresenta a quantidade de $\mathrm{H}_{2}$ adsorvido considerando as isotermas de adsorção total, reversível e irreversível (fortemente adsorvido). Esta última obtida pela diferença entre as duas isotermas anteriores. São apresentados 
também os valores calculados para dispersão metálica e diâmetro médio das partículas metálicas.

Para o catalisador $10 \% \mathrm{Co} / \mathrm{Al}_{2} \mathrm{O}_{3}$ observa-se que a quantidade de $\mathrm{H}_{2}$ adsorvido a $500^{\circ} \mathrm{C}$ é maior do que a $300^{\circ} \mathrm{C}$, considerando-se as isotermas totais e reversíveis. Isto indica que este catalisador não está totalmente reduzido a $300^{\circ} \mathrm{C}$. Realmente, observando os resultados de TPR, verifica-se que este catalisador é mais dificilmente redutível que os demais, requerendo temperaturas mais elevadas.

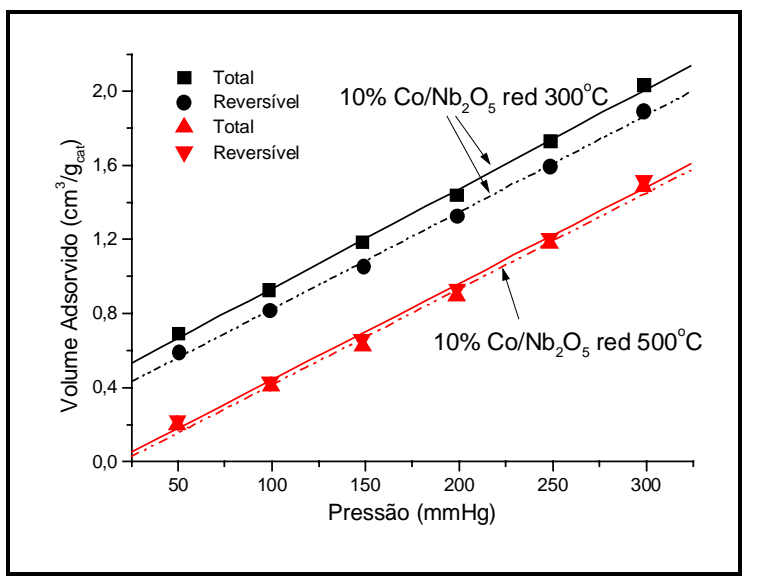

Figura 4 - Quimissorção de $\mathrm{H}_{2}$ para o catalisador $10 \% \quad \mathrm{Co} / \mathrm{Nb}_{2} \mathrm{O}_{5}$ reduzido a $300^{\circ} \mathrm{C}$ e a $500^{\circ} \mathrm{C}$

Tabela 3. Quimissorção de $\mathrm{H}_{2}$, \%Dispersão e diâmetro médio das partículas de cobalto dos catalisadores de $\mathrm{Co} / \mathrm{SiO}_{2}, \mathrm{Co} / \mathrm{Al}_{2} \mathrm{O}_{3}$ e $\mathrm{Co} / \mathrm{Nb}_{2} \mathrm{O}_{5}$.

\begin{tabular}{|c|c|c|c|c|c|c|}
\hline \multirow[t]{2}{*}{ Catalisadores } & \multirow[t]{2}{*}{$\mathrm{T}_{\text {red }}\left({ }^{\circ} \mathrm{C}\right)$} & \multicolumn{3}{|c|}{$\begin{array}{c}\text { Quantidade de } \mathbf{H}_{2} \text { adsorvido } \\
\left(\mu \mathrm{mols} / \mathrm{g}_{\mathrm{cat}}\right)^{\mathrm{a}}\end{array}$} & \multirow{2}{*}{$\begin{array}{c}\text { \%D } \\
\text { Ads. Total }\end{array}$} & \multirow{2}{*}{$\overline{\mathrm{d}}_{\mathrm{P}_{\mathrm{Co}}}(\mathbf{n m})$} \\
\hline & & Total & Rev. & Irrev. & & \\
\hline \multirow{2}{*}{$10 \% \mathrm{Co} / \mathrm{Al}_{2} \mathrm{O}_{3}$} & 300 & 14,7 & 10,7 & 3,6 & 2,89 & 34,5 \\
\hline & 500 & 28,4 & 13,9 & 14,5 & 3,35 & 29,8 \\
\hline \multirow{2}{*}{$5 \% \mathrm{Co} / \mathrm{SiO}_{2}$} & 300 & 28,5 & 19,1 & 9,4 & 10,33 & 9,6 \\
\hline & 500 & 4,6 & 4,5 & 0,1 & 1,08 & 91,9 \\
\hline \multirow{2}{*}{$10 \% \mathrm{Co} / \mathrm{SiO}_{2}$} & 300 & 35,7 & 17,0 & 18,7 & 6,47 & 15,4 \\
\hline & 500 & 21,9 & 17,9 & 4,0 & 2,58 & 38,6 \\
\hline \multirow{2}{*}{$20 \% \mathrm{Co} / \mathrm{SiO}_{2}$} & 300 & 89,7 & 51,7 & 38,0 & 8,13 & 12,3 \\
\hline & 500 & 46,7 & 32,9 & 13,8 & 2,75 & 36,2 \\
\hline \multirow{2}{*}{$10 \% \mathrm{Co} / \mathrm{Nb}_{2} \mathrm{O}_{5}$} & 300 & 17,9 & 13,4 & 4,5 & 3,19 & 31,2 \\
\hline & 500 & $*$ & $*$ & $*$ & - & - \\
\hline
\end{tabular}

a valores calculados por extrapolação das quantidades de $\mathrm{H}_{2}$ adsorvido a pressão igual a zero.

\subsection{REDUÇÃO A TEMPERATURA PROGRAMADA (TPR)}

Os perfis de TPR dos catalisadores estão apresentados na Figura 5. Os perfis de redução obtidos para os suportes não apresentaram picos de redução, enquanto para os catalisadores $\mathrm{Co} / \mathrm{SiO}_{2}, \mathrm{Co} / \mathrm{Al}_{2} \mathrm{O}_{3}$ e $\mathrm{Co} / \mathrm{Nb}_{2} \mathrm{O}_{5}$, calcinados a $400^{\circ} \mathrm{C}$, apresentam dois picos devido a redução $\mathrm{Co}_{3} \mathrm{O}_{4}$ para $\mathrm{CoO}$ e deste para Co.

A redutibilidade dos catalisadores pôde ser avaliada a partir do consumo de $\mathrm{H}_{2}$ medido durante o TPR para a redução do óxido de cobalto: $\mathrm{Co}_{3} \mathrm{O}_{4}+4 \mathrm{H}_{2} \rightarrow 3 \mathrm{Co}$ $+4 \mathrm{H}_{2} \mathrm{O}$. Os resultados apresentados para os catalisadores $10 \% \quad \mathrm{Co} / \mathrm{SiO}_{2}$ e $10 \%$ $\mathrm{Co} / \mathrm{Al}_{2} \mathrm{O}_{3}$ estão de acordo com os obtidos por Lapidus et al. (1991). O perfil de TPR do catalisador $10 \% \mathrm{Co} / \mathrm{Nb}_{2} \mathrm{O}_{5}$, com pico a $525{ }^{\circ} \mathrm{C}$, é semelhante ao apresentado por Silva (1993).

\subsection{TESTE CATALÍTICO}

Catalisadores de cobalto suportados em sílica, alumina e nióbia, com diferentes teores de fase ativa foram testados. A Tabela 4 apresenta os resultados obtidos para os catalisadores com relação à quantidade de carbono formado calculado a 
partir da evolução da conversão do metano durante as 2 primeiras horas de reação.

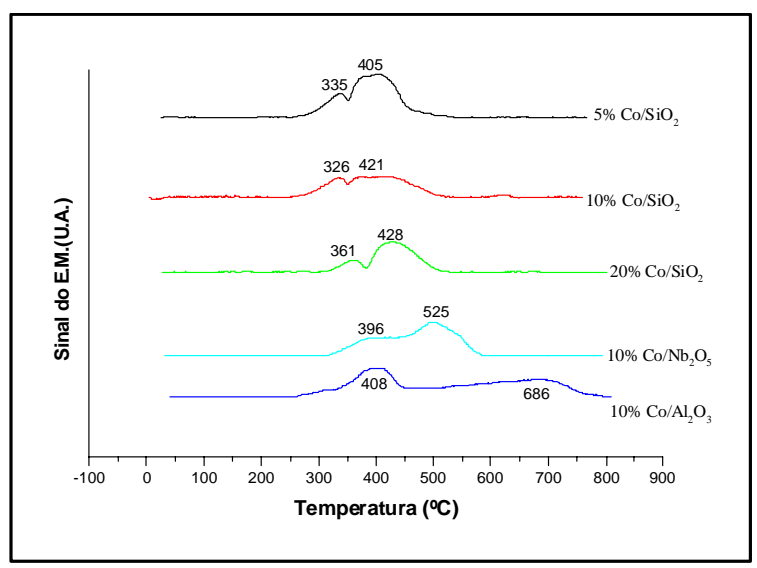

Figura 5 - TPR dos catalisadores de $\mathrm{Co} / \mathrm{SiO}_{2}, \mathrm{Co} / \mathrm{Al}_{2} \mathrm{O}_{3}$ e $\mathrm{Co} / \mathrm{Nb}_{2} \mathrm{O}_{5}$

Tabela 4 - Taxa de formação de depósitos de carbono em catalisadores de cobalto

\begin{tabular}{|c|c|c|}
\hline \multicolumn{3}{|c|}{$\begin{array}{l}\text { Taxa de deposição de C em } \mu \mathrm{mol} \mathrm{C/}(\mu \mathrm{mol} \\
\text { Co.min })^{\mathrm{a}}\end{array}$} \\
\hline Catalisador & $\begin{array}{c}\text { Redução a } \\
300^{\circ} \mathrm{C}\end{array}$ & $\begin{array}{c}\text { Redução a } \\
500^{\circ} \mathrm{C}\end{array}$ \\
\hline $20 \% \mathrm{Co} / \mathrm{SiO}_{2}$ & 2,14 & 6,72 \\
\hline $10 \% \mathrm{Co} / \mathrm{SiO}_{2}$ & 2,73 & 5,61 \\
\hline $10 \% \mathrm{Co} / \mathrm{Nb}_{2} \mathrm{O}_{5}$ & 3,16 & - \\
\hline $10 \% \mathrm{Co} / \mathrm{Al}_{2} \mathrm{O}_{3}$ & 5,67 & 4,94 \\
\hline
\end{tabular}

${ }^{\mathrm{a}}$ Considerando a quimissorção de $\mathrm{H}_{2}$ apresentada na tabela 3.

Os resultados apresentados acima estão de acordo com os obtidos por Boskovic e Smith (1997), que também trabalharam com catalisadores de cobalto suportados em alumina e sílica na decomposição do metano a $450^{\circ} \mathrm{C}$. Esses autores trabalharam com taxas iniciais tendo obtido valores da ordem de 3,63 a 3,73 $\mu \mathrm{mol} \mathrm{C/} \mathrm{( \mu mol} \mathrm{Co.min)} \mathrm{para} \mathrm{os}$ catalisadores de $\mathrm{Co} / \mathrm{Al}_{2} \mathrm{O}_{3}$ reduzidos a $600^{\circ} \mathrm{C}$ e de 1,81 a $2,01 \mu \mathrm{mol} \mathrm{C} /(\mu \mathrm{mol}$ Co.min) para os de $\mathrm{Co} / \mathrm{SiO}_{2}$ reduzidos a $500^{\circ} \mathrm{C}$. Os resultados encontrados no presente artigo parecem mais promissores uma vez que foram superiores aos obtidos por Boskovic (1997).
Pela Tabela 4 verifica-se que para os catalisadores de $\mathrm{Co} / \mathrm{SiO}_{2}$, a redução a $500^{\circ} \mathrm{C}$ propicia uma maior taxa de decomposição de metano e, por conseguinte, maior taxa de formação de depósitos de carbono do que a redução a $300^{\circ} \mathrm{C}$. Isto se explica pelo fato dos catalisadores reduzidos a $300^{\circ} \mathrm{C}$ não estarem totalmente reduzidos após ativação, sendo necessária uma maior temperatura de redução. Realmente, para este catalisador, verificou-se na primeira hora da reação que houve um aumento gradativo da conversão de metano que se explicaria pelo fato do $\mathrm{H}_{2}$ produzido pela decomposição do metano $\left(450^{\circ} \mathrm{C}\right)$ nos sítios metálicos de Co, já presentes no catalisador, estarem reduzindo parte do óxido de cobalto ainda não reduzido. Já o catalisador reduzido a $500^{\circ} \mathrm{C}$ exibiu uma certa estabilidade na conversão do metano durante a reação e superior ao catalisador reduzido a $300^{\circ} \mathrm{C}$ embora possua uma menor dispersão provocada pela sinterização das partículas de cobalto. De qualquer forma, as conversões dos catalisadores reduzidos a $300^{\circ} \mathrm{C}$ mesmo após 2 horas de reação se mantiveram inferiores as dos catalisadores reduzidos a $500^{\circ} \mathrm{C}$. Neste ponto, verifica-se que o tamanho das partículas metálicas exerce um papel fundamental, garantindo maior reatividade aos catalisadores. Os catalisadores que forneceram as maiores conversões foram os que possuíam tamanhos de partículas na faixa de 36-38 nm.

Já o catalisador $10 \% \mathrm{Co} / \mathrm{Al}_{2} \mathrm{O}_{3}$ exibiu um comportamento diferente, apresentando altas taxas de decomposição de metano após redução a $300^{\circ} \mathrm{C}$, embora não totalmente reduzido nessa temperatura. Aqui, também observou-se um aumento gradativo na conversão durante a primeira hora de reação devido a redução do óxido de cobalto pelas mesmas razões mencionadas acima.

Em relação ao catalisador $\mathrm{Co} / \mathrm{Nb}_{2} \mathrm{O}_{5}$ reduzido a $300^{\circ} \mathrm{C}$, observou-se uma certa constância na conversão do metano, apesar 
do catalisador também não estar totalmente reduzido a esta temperatura. Testes de redutibilidade indicaram $66 \%$ de redução. Isto poderia ser explicado em função de estar ocorrendo dois processos simultâneos. A $450^{\circ} \mathrm{C}$, temperatura da reação, o $\mathrm{H}_{2}$ produzido nos sítios metálicos, pela decomposição do metano, além de estar reduzindo as partículas de óxido de cobalto, ainda não reduzidas na ativação à baixa temperatura, estaria reduzindo simultaneamente o suporte, formando espécies $\mathrm{NbO}_{\mathrm{x}}$, móveis, que estariam migrando para a superfície das partículas metálicas, bloqueando-as para a reação. Esta forte interação metal-suporte se caracteriza como o efeito SMSI. Os dois processos descritos estariam competindo e provocando a constância na conversão. Espera-se que após um tempo maior, o efeito SMSI predomine, e a conversão diminua. Isto pode ser justificado pela não reatividade do catalisador após redução a $500^{\circ} \mathrm{C}$, observada durante um teste catalítico realizado, onde não houve decomposição do metano.

\section{CONCLUSÕES}

Os resultados da reação de decomposição de $\mathrm{CH}_{4}$ realizada a $450^{\circ} \mathrm{C}$, em catalisadores de $\mathrm{Co} / \mathrm{SiO}_{2}, \mathrm{Co} / \mathrm{Al}_{2} \mathrm{O}_{3}$ e $\mathrm{Co} / \mathrm{Nb}_{2} \mathrm{O}_{5}$, reduzidos à baixa $\left(300^{\circ} \mathrm{C}\right)$ e à alta temperatura $\left(500^{\circ} \mathrm{C}\right)$ indicaram diferenças distintas.

Os reduzidos a $500^{\circ} \mathrm{C}$ apresentam uma certa constância na conversão, justificada pela redução mais completa durante a ativação à alta temperatura. A conversão do metano ao longo de 2-3 horas de reação se manteve razoavelmente estável, não apresentando sinais de desativação. Todos os catalisadores apresentaram altas taxas de decomposição de metano e, por conseguinte, altas taxas de deposição de carbono.

Os catalisadores reduzidos a $300^{\circ} \mathrm{C}$ apresentam um aumento gradativo na conversão do metano, com uma evolução na produção de $\mathrm{H}_{2}$, que se justifica pela redução de parte do óxido de cobalto pelo $\mathrm{H}_{2}$ produzido pela decomposição do metano nos sítios metálicos de Co, já presentes no início da reação. Testes indicaram que catalisadores de $\mathrm{Co} / \mathrm{SiO}_{2}$ possuem em média $65 \%$ de grau de redução após ativação a esta temperatura. No entanto, para estes catalisadores, mesmo após 2 a 3 horas de reação, as taxas não alcançaram os níveis dos catalisadores reduzidos a $500^{\circ} \mathrm{C}$. O tamanho das partículas de cobalto parecem influir bastante, notando-se altas taxas de deposição de carbono para os que apresentam tamanhos na faixa de 36-38 nm.

Para o catalisador suportado em $\mathrm{Al}_{2} \mathrm{O}_{3}$ reduzido a $300^{\circ} \mathrm{C}$ algumas diferenças podem ser observadas. A conversão parece atingir os patamares obtidos para o reduzido a $500^{\circ} \mathrm{C}$, após 2 horas de reação, produzindo altas taxas de decomposição de metano.

Em relação ao catalisador $\mathrm{Co} / \mathrm{Nb}_{2} \mathrm{O}_{5}$, os resultados são distintos. O catalisador reduzido a $300^{\circ} \mathrm{C}$ apresentou uma constância na conversão do metano ao longo das 2 horas de reação. Tal resultado foi atribuído ao efeito SMSI formado pela redução do suporte pelo $\mathrm{H}_{2}$ gerado nos sítios metálicos pela decomposição do metano e pela redução simultânea das partículas de óxido de cobalto não reduzidas anteriormente, produzindo um efeito de compensação. Testes indicaram que este catalisador possui em média $66 \%$ de grau de redução após ativação a esta temperatura por apenas 3 horas. Após redução à alta temperatura, $500^{\circ} \mathrm{C}$, este catalisador não apresentou reatividade.

\section{REFERÊNCIAS BIBLIOGRÁFICAS}

AVDEEVA L.B.; KOCHUBEY D. I.; SHAIKHUTDINOV S.K. Cobalt Catalysts of Methane Decomposition: Accumulation of the Filamentous Carbon. Applied Catalysis, A., vol. 177, p. 43, 1999. 
BOSKOVIC, G.; SMITH, K. J. Methane Homologation and Reactivity of Carbon Species on Supported Co Catalysts. Catalysis Today, vol.37, p. 25, 1997.

ERMAKOVA, M. A.; ERMAKOV, D. Y.; KUVSHINOV, G. G. Applied Catalysis, A, vol. 201, p. 61, 2000.

FERREIRA-APARÍCIO, P.; GUERRERORUIZ, A.; RODRIGUEZ-RAMOS, I. ; Methane Interaction with Sílica and Alumina Supported Metal Catalysts. Applied Catalysis. A: General, vol. 148, p. 343, 1997.

FIGUEIREDO, J. L.; BERNARDO, C. A.; BAKER, R.T.K.; HUTTINGER, K. J . Carbon Fibers Filaments and Composites. Kluwer Academic Publishers, 1989.

GUARINO, A. P. F.; THOMAZELLI, B. M. O.; PASSOS, F. B.; SILVA, R. R. C. M. O Efeito do Suporte em Catalisadores de $\mathrm{Co} / \mathrm{SiO}_{2}, \mathrm{Co} / \mathrm{Al}_{2} \mathrm{O}_{3}$ e $\mathrm{Co} / \mathrm{Nb}_{2} \mathrm{O}_{5}$ na Decomposição do Metano. In: Anais do $13^{\mathbf{0}}$ Congresso Brasileiro de Catálise, Foz do Iguaçu, Vol. 1, 2005.

GUARINO, A. P. F. Decomposição do Metano em Catalisadores $\mathrm{Co} / \mathrm{SiO}_{2}$, $\mathrm{Co} / \mathrm{Al}_{2} \mathrm{O}_{3}$ e $\mathrm{Co} / \mathrm{Nb}_{2} \mathrm{O}_{5}$. Tese de Mestrado, Universidade Federal Fluminense, 2005.

LAPIDUS, A.; KRYLOVA, A.; KASANSKII, V.; BOROVKOV, V.; ZAITSEV, A. Applied Catalysis, vol. 73, p. 65, 1991.

LI, Y.; ZHANG, B.; TANG, X.; XU Y.; SHEN,W. Hydrogen Production from Methane Decomposition over $\mathrm{Ni} / \mathrm{CeO}_{2}$ Catalysts. Catalysis Communications, vol. 7, p. 380, 2006.

MARTINS, R.; SCHMAL, M.; Methane Activations on Superacidic Catalysts Based on Oxoanion Modified Zirconium Oxide. Applied Catalysis, vol. 208, pp. 143, 2006.

OGIHARA, H.; TAKENAKA, S.; YAMANAKA, I.; TANABE, E.; GENSEKI, A.; OTSUDA, K. Formation of Highly Concentrated Hydrogen Through Methane decomposition over Pd-bases
Alloy Catalysts. Journal of Catalysis, vol. 238, p. 353, 2006.

PINHEIRO, J.P.; SCHOULER, M. C.; DOORYHEE, E. Solid States Communications, vol. 123, p. 161, 2002

SILVA, R. R. C. M.; SCHMAL, M.; FRETY, R.; DALMON J. A. Journal of Chemical Society Faraday Transactions, vol. 89 (21), p. 3975, 1993.

SOLYMOSI, F.; ERDOHELYI, A.; CSERÉNYI, J.; FELVÉGI, A. Activation of $\mathrm{CH}_{4}$ and Its Reaction with $\mathrm{CO}_{2}$ over Supported Rh Catalysts. Journal of Catalysis, vol. 147, pp. 272, 1994.

WU, M.C.; GOODMAN, D. W. J. American Chemical Society, vol. 116, 1364, 1994.

ZADEH, J. S. M.; SMITH, K.J. Journal of Catalysis, vol. 176, pp. 115, 1998.

ZHANG, Y.; SMITH, K. C. H. $\mathrm{CH}_{4}$ Decomposition on Co Catalysts: Effect of Temperature, Dispersion, and the Presence of $\mathrm{H}_{2}$ or $\mathrm{CO}$ in the Feed. Catalysis Today, vol. 77, p. 257, 2002.

ZHANG, Y.; SMITH, J. K. A Kinetic Model of $\mathrm{CH}_{4}$ Decomposition and Filamentous Carbon Formation on Supported Co Catalysts. Journal of Catalysis, 231, p. 354-364, 2005. 